\title{
Competências e Habilidades Cognitivas: Diferentes Definições dos Mesmos Construtos ${ }^{1}$
}

\author{
Ricardo Primi ${ }^{2}$, Acácia A. Angeli dos Santos, \\ Claudette Medeiros Vendramini, Fernanda Taxa, Franz August Muller, \\ Maria de Fátima Lukjanenko e Isabel Silva Sampaio \\ Universidade São Francisco
}

\begin{abstract}
RESUMO - Recentemente, os programas de avaliação implementados pelo governo brasileiro têm chamado a atenção para uma dimensão humana com uma longa tradição de pesquisa na Psicologia, as habilidades cognitivas. Neste artigo procura-se analisar o modelo conceitual de habilidades e competências subjacente ao Exame Nacional do Ensino Médio (ENEM) e compará-lo com os modelos contemporâneos da inteligência humana pela ótica da psicometria e psicologia cognitiva. Embora empreguem terminologias diferentes, os modelos referem-se a dimensões comuns da capacidade humana, nomeadamente a inteligência fluida e cristalizada mas ainda faltam estudos de validade de construto para evidenciar com mais clareza o que o ENEM avalia.
\end{abstract}

Palavras-chave: Exame Nacional do Ensino Médio; avaliação da inteligência; habilidades; competências.

\section{Cognitive Abilities and Competencies: Distinct Terms for the Same Constructs}

\begin{abstract}
Recently, educational assessment systems implemented by the Brazilian government have called attention to the human cognitive abilities that have been under study by psychologists since a long time ago. This work analyze the conceptual model of abilities and competencies that underlies the National Exam of High School Education comparing it with contemporary models of human intelligence from psychometrics and cognitive psychology traditions. Although the models use distinct terms they are referring to similar constructs namely fluid and crystallized intelligence, nevertheless construct validity studies are necessary to investigate more clearly what construct the test measures.
\end{abstract}

Key words: National Exam of High School Education; intelligence assessment; cognitive abilities and competencies.

Recentemente, os programas de avaliação implementados pelo governo brasileiro, tais como o Sistema de Avaliação do Ensino Básico (SAEB), o Exame Nacional do Ensino Médio (ENEM) e o Exame Nacional de Cursos (ENC, Instituto Nacional de Estudos e Pesquisas Educacionais [INEP], 1997, 1998a, 1998b e 1999), têm chamado a atenção para uma dimensão humana com uma longa tradição de pesquisa na Psicologia: as habilidades cognitivas (Ackerman, Kyllonen \& Roberts, 1999; Almeida, 1988; Flanagan, Genshaft \& Harrison, 1997; Hunt, 1996; 1999; Sternberg, 1981).

Pode-se observar que, no trabalho das comissões compostas para elaboração dessas provas, os especialistas definem um conjunto de habilidades consideradas essenciais e, a partir delas, constróem os instrumentos de avaliação. Um fato que geralmente ocorre a partir de implementação de sistemas de avaliação nacionais, como esses, é que as insti-

1 Este estudo é parte de uma pesquisa em andamento cujo tema é a Avaliação das Habilidades e Aprendizagem em Universitários financiada pelo Programa de Estímulo à Pesquisa Científica (PEPCI) da Universidade São Francisco.

2 Endereço: Ricardo Primi, Universidade São Francisco - Programa de Estudos Pós-Graduados em Psicologia, Laboratório de Avaliação Psicológica e Educacional, Rua Alexandre Rodrigues Barbosa, 45, CEP 13251-900, Itatiba - SP. E-mail: labape@ saofrancisco.edu.br. tuições educacionais passam a orientar o desenvolvimento das habilidades e competências consideradas para a avaliação. Portanto, sua definição é de fundamental importância, considerando o impacto que podem produzir nos projetos pedagógicos das universidades.

O objetivo deste artigo é examinar as definições de competências e habilidades propostas pelo ENEM (INEP, 1999) à luz de concepções recentes, resultantes dos estudos sobre a inteligência, principalmente os das abordagens psicométrica e do processamento humano de informação. Será apresentada uma síntese das definições de habilidades e competências propostas pelo ENEM; em seguida, a dos modelos mais recentes da inteligência e, finalmente, serão examinadas as semelhanças e diferenças entre ambas.

\section{Competências e Habilidades no ENEM}

O ENEM estrutura-se a partir de uma matriz de competências e habilidades que fundamenta a construção dos itens e informa como os autores entendem a inteligência e quais, entre as suas dimensões, devem ser privilegiadas pelo Ensino Médio. Segundo os autores, ela corresponde às "possibilidades totais da cognição humana na fase de desenvolvimento próprio aos participantes do ENEM" (INEP, 1999, p. 9). As definições gerais de competências e habilidades são: 


\section{R. Primi e cols.}

Tabela 1. Matriz de Competências e Habilidades do ENEM adaptada do Instituto Nacional de Estudos e Pesquisas Educacionais (1999).

\begin{tabular}{|c|c|c|c|c|c|c|c|c|c|c|c|c|c|c|c|c|c|c|c|c|c|c|}
\hline \multirow{2}{*}{\multicolumn{2}{|c|}{ Competências }} & \multicolumn{21}{|c|}{$\begin{array}{c}\text { Habilidades } \\
\text { (ver INEP, } 1999 \text { para uma descrição detalhada de cada habilidade) }\end{array}$} \\
\hline & & 1 & 2 & 3 & 4 & 5 & 6 & 7 & 8 & 9 & 10 & 11 & 12 & 13 & 14 & 15 & 16 & 17 & 18 & 19 & 20 & 21 \\
\hline I. & $\begin{array}{l}\text { Dominar linguagens } \\
\text { Dominar a norma culta da Língua Portuguesa e fazer } \\
\text { uso das linguagens matemática, artística e científica. }\end{array}$ & & & & & & & & & & & & & & & & & & & & & \\
\hline II. & $\begin{array}{l}\text { Compreender fenômenos } \\
\text { Construir e aplicar conceitos das várias áreas do } \\
\text { conhecimento para a compreensão de fenômenos } \\
\text { naturais, de processos histórico-geográficos, da } \\
\text { produção tecnológica e das manifestações artísticas. }\end{array}$ & & & & & & & & & & & & & & & & & & & & & \\
\hline III. & $\begin{array}{l}\text { Enfrentar situações-problema } \\
\text { Selecionar, organizar, relacionar, interpretar dados e } \\
\text { informações representados de diferentes formas, para } \\
\text { tomar decisões e enfrentar situações-problema. }\end{array}$ & & & & & & & & & & & & & & & & & & & & & \\
\hline IV. & $\begin{array}{l}\text { Construir argumentações } \\
\text { Relacionar informações, representadas em diferentes } \\
\text { formas, e conhecimentos disponíveis em situações } \\
\text { concretas para construir argumentação consistente. }\end{array}$ & & & & & & & & & & & & & & & & & & & & & \\
\hline & $\begin{array}{l}\text { Elaborar propostas } \\
\text { Recorrer aos conhecimentos desenvolvidos na escola } \\
\text { para a elaboração de propostas de intervenção } \\
\text { solidária na realidade, respeitando os valores } \\
\text { humanos e considerando a diversidade socio-cultural. }\end{array}$ & & & & & & & & & & & & & & & & & & & & & \\
\hline
\end{tabular}

Competências são as modalidades estruturais da inteligência, ou melhor, ações e operações que utilizamos para estabelecer relações com e entre objetos, situações, fenômenos e pessoas que desejamos conhecer. As habilidades decorrem das competências adquiridas e referem-se ao plano imediato do 'saber fazer'. Por meio das ações e operações, as habilidades aperfeiçoam-se e articulam-se, possibilitando nova reorganização das competências (INEP, 1999, p.7).

São propostas cinco competências gerais, descritas na Tabela 1, referidas pelos nomes: dominar linguagens, compreender fenômenos, enfrentar situações-problema, construir argumentações e elaborar propostas. Em um nível mais específico, são definidas 21 habilidades $^{3}$, que na Tabela 1 são indicadas pelos seus números, cuja especificidade refere-se à delimitação de conteúdos, tais como, utilização de recursos naturais - conhecimento associado principalmente às ciências físicas e biológicas, (Habilidades 7, 8, 9 e 17), conhecimento dos processos vitais de um ponto de vista sistêmico (Habilidades 10, 11, 12, 13 e 16), conceitos matemáticos e estatísticos (Habilidades 14 e 15), processos sociais econômicos histórico-geográficos (Habilidades 18, 20 e 21) entre outros (Menezes, Gualtieri, Guimarães, Lisboa \& Kawamura, 1999).

As 21 habilidades orientam o trabalho de especialistas que constróem um banco de itens, posteriormente analisados quanto à adequação aos propósitos da matriz de competências e de orientações gerais do ENEM (Fini, Charnet, Talim \& Moraes, 1999) e quanto à adequação do ponto de vista psicométrico, necessitando para isso de uma pré

3 Ver INEP (1999) sobre a descrição destas habilidades. testagem, para que sejam estabelecidos os níveis de dificuldade, de discriminação, e a possibilidade de acertos ao acaso, utilizando-se os métodos de análise da Teoria de Resposta ao Item (Hambleton \& Swaminatham,1985). A partir dessas análises selecionam-se três itens para cada habilidade, gerando-se um instrumento com 63 itens. Na prova de 1999, por exemplo, os itens finais foram selecionados de um conjunto de aproximadamente 500 itens, levando-se em consideração, simultaneamente, sua adequação ao conteúdo da matriz de competências e habilidades e sua qualidade psicométrica. Algumas características gerais a distinguem das provas tradicionalmente usadas em vestibulares.

Um primeiro aspecto refere-se à mudança de ênfase de avaliação de conteúdos memorizados para a avaliação de processos gerais de raciocínio. Como afirma Macedo (1999):

Até há pouco tempo, a grande questão escolar era a aprendizagem - exclusiva ou preferencial - de conceitos. Estávamos dominados pela visão de que conhecer é acumular conceitos; ser inteligente implicava articular logicamente grandes idéias, estar informado sobre grandes conhecimentos ... Este tipo de aula, insisto, continua tendo lugar, mas cada vez mais torna-se necessário também o domínio de um conteúdo chamado 'procedimental', ou seja da ordem do saber como fazer. Vivemos em uma sociedade cada vez mais tecnológica, em que o problema nem sempre está na falta de informações, pois o computador tem cada vez mais o poder de processá-las, guardálas ou atualizá-las. A questão está em encontrar, interpretar essas informações, na busca de solução de nossos problemas (p.8).

Tal ênfase está refletida numa importante característica dos itens do ENEM: o fornecimento, nas questões, da maioria das informações necessárias à resolução do problema 
proposto. Uma questão tradicional centrada na memória requer, para resolvê-la, que o aluno aplique conhecimentos e procedimentos previamente adquiridos. Ele só conseguirá responder se tiver aprendido e conseguir lembrá-los no momento da avaliação (fórmulas, definições, fatos, etc.). Por outro lado, uma questão centrada mais no raciocínio, requer a interpretação e relacionamento das informações disponíveis nas questões. A resposta do aluno a uma questão deste tipo depende menos da recordação de conhecimentos prévios e mais da recombinação de conhecimentos já existentes de maneira nova. Com isso não queremos dizer que as provas prescindam dos conhecimentos adquiridos no passado. Eles sempre terão uma influência no processo de resolução, o que queremos enfatizar é que, em comparação com uma prova tradicional, a importância desse conhecimento é menor no ENEM.

Uma segunda característica dos itens do ENEM referese à utilização de situações-problema contextuadas (Macedo, 1999; Machado, 1999). Por exemplo, nas provas tradicionais de avaliação do conhecimento pode-se fazer uma questão envolvendo conhecimentos de química perguntando-se simplesmente se o aluno conhece como os indicadores base reagem em soluções aquosas com diferentes pHs. No ENEM, por outro lado, tal conhecimento é apresentado em um contexto em que é necessário tomar uma decisão se houve falsificação de água mineral (Questão 6, prova branca, ENEM 1999). Portanto, o conhecimento deve ser usado como instrumento para a resolução de um problema da experiência cotidiana.

Uma terceira característica importante do ENEM referese à interdisciplinaridade das questões (Machado, 1999). Na maioria delas, os autores procuraram incluir conhecimentos de mais de uma área. Essa inter-relação pode ser percebida na matriz de habilidades. Nela, não aparece claramente a distinção formulada entre as áreas de conhecimento, como habitualmente é visto nas provas de processos seletivos; em vez disso vê-se uma combinação de áreas (por exemplo, as Habilidades 7, 8, 9 e 17, envolvendo conhecimentos de Física, Biologia, Economia, Geografia, História e Ética; Menezes e cols., 1999).

A nosso ver são estas três importantes características que distinguem o ENEM e o tornam superior às provas habitualmente usadas nos vestibulares até então.

\section{Competências e Habilidades na Psicometria e na Psicologia Cognitiva}

Os modelos explicativos da inteligência têm sido classificados em três grandes correntes, a psicométrica (ou fatorial), a desenvolvimentalista, e a da abordagem do processamento humano de informação (Almeida, 1988, 1994; Sternberg, 1977, 1979, 1980, 1981, 1983, 1986). A psicométrica concentra-se em definir as estruturas da inteligência e sua organização, o que a torna vulnerável à crítica de que só investiga o produto (diferenças individuais nos testes de inteligência) e não o processo cognitivo que leva ao produto. A desenvolvimentalista, baseada na ótica de Piaget e de Vigotsky, procu- ra definir as estruturas da inteligência e sua dinâmica ao longo do desenvolvimento, trazendo avanços significativos ao procurar descrever o processamento cognitivo e relacionálo aos diferentes estágios de desenvolvimento. Mas, a mais marcante reação à abordagem psicométrica foi a do processamento da informação (Hunt, 1980; Newell \& Simon, 1972; Sternberg, 1977), que a partir da década de 60 , deu origem a um grande volume de estudos investigando detalhadamente os processos cognitivos envolvidos na resolução dos testes tradicionais usados pela psicometria (Goldman \& Pellegrino, 1984; Pellegrino \& Glaser, 1979; Pellegrino \& Lyon, 1979; Primi, 1995). Tal abordagem vem gradualmente se integrando aos estudos da neurologia dando origem à neurociência cognitiva (Hunt, 1999).

O ENEM fundamenta-se claramente nos pressupostos da corrente desenvolvimentalista de Piaget, a partir, portanto, de uma corrente européia de pensamento sobre a inteligência (Macedo, 1999; Machado, 1999; Perrenoud, 1997).

Neste artigo procuraremos traçar as semelhanças e diferenças dos conceitos de competências e habilidades do ENEM com as abordagens psicométrica e do processamento de informação, as quais representam uma corrente prioritariamente norte-americana. Esta distinção é importante pois, embora tratem do mesmo fenômeno, as duas correntes adotam terminologias diferentes. Em seguida, apresentaremos uma síntese de alguns trabalhos importantes da área, considerados representativos dos modelos atuais que procuram definir a inteligência humana para posteriormente discutirmos as semelhanças e diferenças entre estes modelos e as concepções de inteligência adotadas no ENEM.

\section{A Teoria dos Três Estratos}

Ao se tratar da visão psicométrica da inteligência há de se considerar que provavelmente a maioria destes estudos baseou-se na análise fatorial, um método de análise de estruturas de covariância. Como escreve Carroll (1993):

a idéia básica, embora algo simplificada, é que quando se encontram duas variáveis, representando diferenças entre indivíduos, substancialmente correlacionadas, há a possibilidade de que uma única habilidade cognitiva ou um conjunto unitário de habilidades cognitivas esteja subjacente a estas variáveis, no entanto quando duas variáveis não estão correlacionadas é provável que duas habilidades estejam subjacentes a elas (p. 5).

A grande maioria dos estudos psicométricos da inteligência, nas primeiras sete décadas do século $\mathrm{XX}$, aplicava baterias de testes e empregava a análise fatorial buscando descobrir como estes testes se correlacionavam e definia, a partir dos agrupamentos formados, as estruturas da inteligência (fatores, habilidades, aptidões).

O principal ponto de controvérsia e tensão na abordagem psicométrica refere-se às discussões entre os modelos que interpretam as correlações como indicadoras da existência de uma única habilidade geral (fator g, Spearman, 1927) e os modelos que explicam as correlações pela exis- 


\section{R. Primi e cols.}

Tabela 2. Modelo de Três Estratos de John B. Carroll adaptado de Carroll (1993).

\begin{tabular}{|c|c|c|c|}
\hline Estrato III & Estrato II & & Estrato I \\
\hline & & & Exemplos de fatores mais significativos. \\
\hline \multirow[t]{10}{*}{ Fator $\mathrm{g}$} & $\begin{array}{l}\text { Inteligência Fluida } \\
\text { Gf }\end{array}$ & $\begin{array}{l}\text { Habilidade de raciocínio em situações novas minimamente } \\
\text { dependentes de conhecimentos adquiridos. }\end{array}$ & $\begin{array}{l}\text { Raciocínio Seqüencial } \\
\text { Indução }\end{array}$ \\
\hline & & $\begin{array}{l}\text { Capacidade de resolver problemas novos, relacionar idéias, } \\
\text { induzir conceitos abstratos, compreender implicações. }\end{array}$ & Raciocínio Quantitativo \\
\hline & $\begin{array}{l}\text { Inteligência } \\
\text { Cristalizada }\end{array}$ & $\begin{array}{l}\text { Habilidade associada à extensão e profundidade dos } \\
\text { conhecimentos adquiridos de uma determinada cultura. }\end{array}$ & $\begin{array}{l}\text { Desenvolvimento Lingüístico } \\
\text { Conhecimento Léxico }\end{array}$ \\
\hline & Gc & $\begin{array}{l}\text { Habilidade de raciocínio adquirida pelo investimento da } \\
\text { capacidade geral em experiências de aprendizagem. }\end{array}$ & Compreensão em Leitura \\
\hline & $\begin{array}{l}\text { Memória e } \\
\text { Aprendizagem } \\
\text { Gsm }\end{array}$ & $\begin{array}{l}\text { Habilidade associada à manutenção de informações na } \\
\text { consciência por um curto espaço de tempo para poder } \\
\text { recuperá-las logo em seguida. }\end{array}$ & $\begin{array}{l}\text { Extensão da memória } \\
\text { Memória associativa } \\
\text { Memória Visual }\end{array}$ \\
\hline & & $\begin{array}{l}\text { Habilidade também associada à quantidade de informação } \\
\text { retida após exposição à uma situação de aprendizagem } \\
\text { (geralmente conteúdos simples). }\end{array}$ & $\begin{array}{l}\text { (Obs: Carroll [993] sugere que as evidências } \\
\text { que encontrou não permitem apresentar uma } \\
\text { definição precisa deste fator). }\end{array}$ \\
\hline & $\begin{array}{l}\text { Percepção Visual } \\
\text { Gv }\end{array}$ & $\begin{array}{l}\text { Habilidade de gerar, reter e manipular imagens visuais } \\
\text { abstratas. }\end{array}$ & $\begin{array}{l}\text { Visualização } \\
\text { Relações espaciais } \\
\text { Velocidade de fechamento }\end{array}$ \\
\hline & $\begin{array}{l}\text { Recepção Auditiva } \\
\text { Ga }\end{array}$ & $\begin{array}{l}\text { Habilidade associada à percepção e discriminação de } \\
\text { padrões sonoros ( incluindo a linguagem oral) } \\
\text { particularmente quando apresentados em contextos mais } \\
\text { complexos envolvendo distorções ou estruturas musicais } \\
\text { complexas. }\end{array}$ & $\begin{array}{l}\text { Acuidade auditiva } \\
\text { Discriminação da linguagem oral } \\
\text { Discriminação e julgamento padrões tonais } \\
\text { musicais }\end{array}$ \\
\hline & $\begin{array}{l}\text { Habilidade de } \\
\text { Recuperação } \\
\text { Glr }\end{array}$ & $\begin{array}{l}\text { Habilidade associada à extensão e fluência que itens de } \\
\text { informação ou conceitos são recuperados da memória de } \\
\text { longo prazo por associação }\end{array}$ & $\begin{array}{l}\text { Fluência de idéias } \\
\text { Facilidade de recordação de nomes } \\
\text { Fluência de associações } \\
\text { Originalidade/Criatividade }\end{array}$ \\
\hline & $\begin{array}{l}\text { Velocidade de } \\
\text { Processamento } \\
\text { Cognitivo } \\
\text { Gs }\end{array}$ & $\begin{array}{l}\text { Habilidade associada à taxa de rapidez de processamento } \\
\text { cogntivo em tarefas cognitivas simples. }\end{array}$ & $\begin{array}{l}\text { Velocidade perceptual } \\
\text { Tempo de reação simples } \\
\text { Velocidade de processamento semântico }\end{array}$ \\
\hline
\end{tabular}

tência de várias habilidades (aptidões primárias, Thurstone, 1938; Guilford, 1967; Horn, 1991; Horn \& Noll, 1997). Até há pouco tempo existia a impressão de que a psicometria ainda não tinha produzido um modelo para inteligência humana de relativo consenso entre os psicólogos. Só depois da década de 90, um modelo hierárquico chamado Teoria de Três Estratos de Carroll (1993) passou a ser considerado relativamente consensual dentro da psicometria (Flanagan \& McGrew, 1997; Marañon \& Pueyo, 1999; McGrew, 1998).

Carroll (1993) desenvolveu uma meta análise de quase todos os principais estudos fatoriais sobre a inteligência publicados de 1920 até 1980 . Foram reunidos 1500 artigos, a partir dos quais 450 matrizes de correlações foram selecionadas e reanalisadas utilizando-se procedimentos metodológicos mais adequados de análise fatorial (análise fatorial hierárquica, Schmid \& Leiman, 1957). Este estudo resultou no modelo hierárquico de três estratos resumido na Tabela 2 , que representa os níveis de generalidade das habilidades. Os fatores do primeiro estrato são mais específicos e geralmente associados às tarefas existentes nos testes de inteligência, que se organizam em sete fatores de segunda ordem, mais abrangentes (broad factors). Por sua vez, estes fatores de segunda ordem se relacionam entre si dando origem ao Estrato III próximo ao que Spearman (1927) chamou de inteligência geral.

Na Tabela 2 apresentamos cada estrato em uma coluna. $\mathrm{Na}$ terceira coluna, apresentamos exemplos de fatores pri- mários de cada fator abrangente, que foram escolhidos por serem os que apresentam maior associação com os fatores abrangentes. Na segunda coluna, apresentamos um resumo dos significados dos fatores abrangentes, sendo que a ordem em que aparecem indica a associação com o fator geral. Assim, verifica-se que a inteligência fluida é a mais próxima do fator $\mathrm{g}$ e a velocidade de processamento cognitivo, a mais distante. Como afirma Carroll (1993): "há evidências abundantes de um fator geral de inteligência que domina os fatores ou variáveis que enfatizam um nível de complexidade elevada possível de ser dominado em tarefas de indução, raciocínio, visualização e compreensão lingüística" (p. 624).

Como pode ser observado, Carroll (1993) chamou os fatores de habilidades cognitivas (abilities). Portanto, o significado atribuído ao termo "habilidade" difere substancialmente do significado que no ENEM se atribui às habilidades. O significado que o autor atribui às habilidades aproxima-se mais do conceito de "competências" adotado no ENEM, embora o número e a natureza destas estruturas sejam diferentes nos dois modelos (Macedo, 1999; Perrenoud, 1997). No entanto, antes de estabelecermos tais relações é pertinente uma discussão de como estes conceitos têm sido definidos pela Psicologia, particularmente pela escola norte-americana.

Carroll (1993) considerou o termo "habilidade" de maneira neutra e geral referindo-se às "variações individuais nos potenciais para realização em uma classe definida de 
tarefas" (p. 16). As sete habilidades abrangentes definem, mais precisamente, os tipos de tarefas para as quais as pessoas revelam possuir potencial diferenciado. Distingue, ainda, o conceito de habilidade do conceito de aptidão afirmando que: "à medida em que as habilidades cognitivas são relativamente estáveis e relativamente resistentes às tentativas de mudança por meio de experiências educacionais ou de treinamento, e ao mesmo tempo, são preditoras de sucessos futuros, elas são muitas vezes consideradas aptidões" (Carroll, 1993; p. 16). Portanto, a definição de habilidade cognitiva não necessariamente compartilha a noção de estabilidade temporal, e portanto, de influência genética implícita no conceito de aptidão. ${ }^{4}$

Mayer e Salovey (1998) fazem uma distinção importante entre habilidade (referindo-as como aptidões) e competência. Os autores argumentam que habilidade representa o potencial que se expressa, concretamente, em realizações ou desempenhos, envolvendo a apresentação de respostas corretas para problemas e conhecimento de determinado conteúdo etc. A competência, nesta concepção, indicaria um nível padronizado de realização, o que implicaria em dizer que a realização atingiu um determinado nível. A nosso ver, esta distinção é melhor formulada, mais oportuna e abrangente, pois relaciona os conceitos: habilidade, conteúdo e nível de realização. A habilidade, no sentido utilizado por Carroll (1993), pressupõe a idéia de potencial de realização, ou seja, a existência de uma relativa facilidade em lidar com informações e com problemas de uma determinada classe ou conteúdo. O investimento deste potencial em experiências de aprendizagem pode conduzir à maestria, que envolve um conhecimento organizado sobre um determinado tema. A idéia de maestria implica que a realização atingiu um determinado nível a partir do qual se diz que uma competência foi adquirida.

Dessa forma, é possível pensar que a habilidade não necessariamente implica em competência. A habilidade indica facilidade em lidar com um tipo de informação e para que se transforme em competência será necessário investimento em experiências de aprendizagem. No entanto, se não houver investimento, não haverá competência, mesmo que a pessoa tenha habilidade em determinada área. Considerando o mesmo montante de experiência, com a mesma qualidade, duas pessoas com habilidades diferentes diferirão na facilidade com que irão adquirir a maestria, ou que se tornarão competentes em determinado tema. Dentro deste enfoque, existem pelo menos três fatores associados ao desenvolvimento de competência: habilidade, montante de investimento e qualidade das experiências de aprendizagem.

4 Talvez na língua portuguesa o termo habilidade tenha um significado mais restrito daquele que é atribuído ao termo "abilities" na língua inglesa. Diante disso pode-se argumentar que o termo mais adequado para traduzi-lo seria "capacidade". No entanto, por uma questão de continuidade com a terminologia usada em trabalhos anteriores utilizaremos o termo habilidade entendendo-o como sinônimo de capacidade.

\section{O Modelo RADEX}

Um segundo modelo explicativo da inteligência, importante de ser considerado, pois, como afirma Sternberg (1981), representa o último estágio evolucionário das teorias fatoriais, é o modelo RADEX (sigla criada a partir de Radial Expansion of Complexity). Este modelo foi criado por Guttman (1954) e designa um método diferenciado baseado na análise multidimensional para a investigação da estrutura de organização das habilidades (Kruskal, 1964). Na década de 80, Snow, Kyllonen e Marshalek (1984), da Universidade de Stanford, aplicaram este método na reanálise de estudos clássicos da inteligência, buscando construir um mapa representando a organização das habilidades e suas relações com a aprendizagem. Neste estudo os autores compararam o RADEX com os modelos hierárquicos da inteligência (a teoria dos três estratos é a versão mais atualizada deles) e concluíram que eles se complementavam e que a representação multidimensional revelava novos aspectos importantes, permitindo integrar informações sobre a estrutura da inteligência (abordagem psicométrica) com informações sobre o processamento da informação.

Snow e cols. (1984) concebem o sistema cognitivo humano como um banco de estruturas organizadas de conhecimento e de processamento das informações, também chamado de programas de performance. Referem-se às informações contidas nesse banco como componentes de resposta, que não precisam ser, necessariamente, unidades simples (vinculadas a um conteúdo único), mas também, e talvez prioritariamente, cadeias organizadas, incluindo unidades mais complexas (que combinam conteúdos e processos cognitivos). Para eles uma determinada situação-problema constitui-se numa condição que requer que determinados componentes sejam aplicados, podendo resultar em uma resposta externa e/ou uma mudança no sistema cognitivo interno. A resolução de um problema implica na aplicação (escolha) de conjuntos de componentes específicos, que devem ser arranjados ou organizados (assembly of components) estrategicamente. Assim, os problemas se distinguem em função dos componentes, do arranjo entre eles e das adaptações necessárias destes arranjos à medida em que a pessoa procede nos passos da resolução. Do mesmo modo, as pessoas diferem entre si, em função de quais componentes, arranjos e adaptações podem produzir para resolver um determinado problema. Quando o desempenho em duas tarefas está correlacionado, pode-se inferir que estas tarefas demandam amostras de componentes, arranjos e adaptações semelhantes.

Marshalek, Lohman e Snow (1983) e Snow e cols. (1984), seguindo o procedimento de Guttman (1954), analisaram as intercorrelações entre testes psicométricos por uma análise multidimensional, na qual os testes foram representados como pontos em um espaço bidimensional. A localização relativa dos pontos está associada às correlações entre eles, isto é, pontos de testes altamente correlacionados tendem a aparecer agrupados no espaço. Ao contrário, pontos de testes não correlacionados tendem a aparecer separados. A aná- 
lise do espaço resultante indicou a existência de dois padrões importantes: (a) testes com mesmo conteúdo se agrupam numa reta, ordenando-se dos mais simples para os mais complexos. Retas formadas por conteúdos distintos convergem para um ponto central, representando uma maior complexidade; (b) testes com mesmo nível de complexidade se agrupam equiidistantes do centro, formando um círculo, diferindo no conteúdo. Guttman (1954) chamou tais padrões, respectivamente, de simplex e circumplex e a organização simultânea destes padrões de radex. A Figura 1 apresenta uma configuração radex dos subtestes do WISC (Escala de Inteligência Wechsler para Crianças [WISC], Wechsler, 1964) e as Matrizes Progressivas de Raven (J. Raven, J. C. Raven \& Court, 1998), cuja figura foi criada a partir das discussões de Snow e cols.

O plano é dividido em três facetas, visual-espacial, quantitativo-numérico e verbal; representando a dimensão conteúdo (circumplex). Além disso, as distâncias periferia-centro indicam a dimensão da complexidade (simplex). É importante frisar algumas características a respeito deste gráfico.

Geometricamente, sabemos que o centro da circunferência é o ponto que possui uma distância média menor de todos os outros, portanto, os testes que aparecem perto do centro são os que apresentam maior correlação com todos os outros. Inversamente, testes que aparecem na periferia tendem a exibir menor correlação com os outros. Como pode ser observado, esta configuração confirma a relação encontrada por Carroll (1993), na qual os fatores abrangentes das inteligências fluida, cristalizada e percepção visual são os que mais se correlacionam com o fator $\mathrm{g}$ (centro da circunferência).

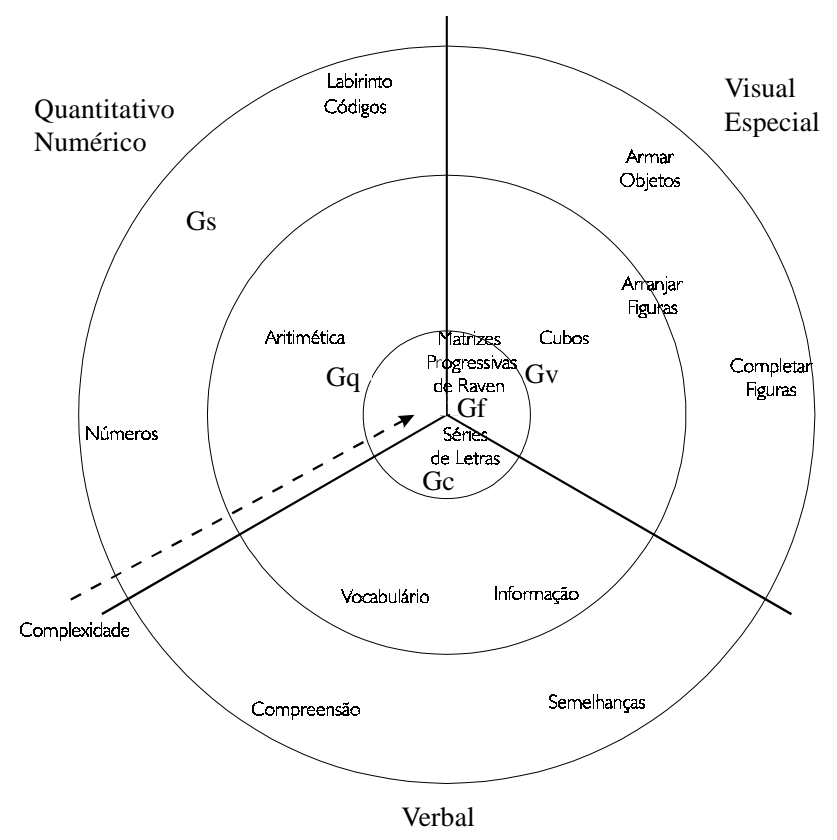

Figura 1. Modelo RADEX de representação da Inteligência Baseado em Snow, Kyllonen \& Marshalek (1984)
Os testes que aparecem no centro da circunferência incluem problemas de complexidade maior do que os testes que aparecem na periferia e, geralmente, são testes que envolvem problemas novos de inferência de relações. A análise comparativa dos processos cognitivos envolvidos na resolução destes testes, em relação aos da periferia, indica que eles requerem processos mais gerais de raciocínio. Estes processos incluem funções executivas de arranjo de componentes de resposta em estratégias de solução de problema, isto é, a reorganização de esquemas prévios em novas estratégias ou programas de performance para a resolução do problema. Envolve também o controle do processamento (metacognição) incluindo o monitoramento e a adaptação flexível desses programas a medida que o problema é resolvido (Bethell-Fox, Lohman \& Snow,1984). Ao contrário, os testes que se localizam na periferia são resolvidos por programas de performance mais automáticos que não requerem um processo de controle e adaptação tão atento.

Estes estudos ressaltam a importância da distinção entre duas habilidades com longa tradição na psicometria, as inteligências fluida e cristalizada. A este respeito, Snow e cols. (1984) afirmam:

O sistema cognitivo humano deve de alguma maneira se engajar em adquirir, reter e reutilizar conceitos e procedimentos que irão ajudá-lo em novas situações-problema e de aprendizagem. Mas o sistema deve também se engajar em adaptar estes conceitos cristalizados previamente e organizar novos conceitos porque as novas tarefas a serem enfrentadas diferem apreciavelmente daquelas experienciadas no passado (p. 98).

Com relação ao conceito de habilidade a concepção deste grupo de pesquisadores difere também da de Carroll (1993), tendo em vista que eles têm utilizado o termo aptidão, sem se referir ao problema de estabilidade ou não do construto. Snow (1992) define aptidão como uma predisposição das pessoas em emitir respostas diferenciais à determinada situação ou classe de situações, significando um estado inicial que irá influenciar o desenvolvimento posterior, dadas determinadas condições. A idéia principal destes autores, que culminou na denominação Aptitude-TreatmentInteraction (ATI), é a de que uma aptidão sempre interage com uma situação, por exemplo, uma alta capacidade de inteligência fluida significa uma pré-disposição de bom desempenho em situações relativamente incompletas, pouco estruturadas, nas quais a pessoa poderá ter um papel mais ativo e independente na organização das informações, que não necessariamente significará a mesma prédisposição em situações mais estruturadas e muito dependentes da aplicação de conhecimentos prévios (Snow, 1989). Portanto, as pessoas possuem aptidões ou habilidades, como referidas neste texto, para se desempenhar em determinadas situações específicas.

\section{Competências e Habilidades: Uma Síntese}

Resumindo, nas abordagens da inteligência apresentadas anteriormente, duas estruturas são tratadas como funda- 
mentais: a inteligência cristalizada (que prioriza o conhecimento) e inteligência fluida (que prioriza o raciocínio). A primeira refere-se à extensão e profundidade das informações adquiridas via escolarização, que geralmente são usadas na resolução de problemas semelhantes aos que se aprendeu no passado, ou ao "estoque" acumulado de conhecimentos, isto é, esquemas organizados de informações sobre áreas específicas do conhecimento. A segunda refere-se à capacidade de processamento cognitivo, isto é, à capacidade geral de processar informações (por exemplo, relacionar idéias complexas, formar conceitos abstratos, derivar implicações lógicas a partir de regras gerais) ou às operações mentais realizadas quando se resolvem problemas relativamente novos, para os quais existem poucos conhecimentos previamente memorizados. Esta capacidade implica na criação de estratégias a partir da organização das informações disponíveis na situação e da reorganização de esquemas disponíveis em nosso estoque de conhecimentos (Ackerman, 1996; Ackerman \& Heggestad, 1997; Ackerman, Kyllonen \& Roberts, 1999).

Na definição de competência de Perrenoud (1997), um dos grandes inspiradores dos criadores do ENEM, observase esta distinção embora o autor não utilize a mesma nomenclatura e nem se baseie nas referências aqui apresentadas. Ele considera que os dois aspectos da competência são: o conhecimento e a capacidade de mobilização do conhecimento. Competência significa, simultaneamente, a erudição e a capacidade de mobilização do conhecimento frente à uma situação problema. Como afirma,

um especialista é competente porque simultaneamente: (a) domina, com muita rapidez e segurança, as situações mais comuns, por ter à sua disposição esquemas complexos que podem entrar imediata e automaticamente em ação, sem vacilação ou reflexão real; (b) é capaz de, com um esforço razoável de reflexão, coordenar e diferenciar rapidamente seus esquemas de ação e seus conhecimentos para enfrentar situações inéditas (p. 27).

Tal distinção assemelha-se exatamente aos conceitos de inteligência cristalizada e fluida respectivamente. Como sabemos, grande parte dos processos seletivos no Brasil têm tradicionalmente privilegiado a noção de inteligência como capacidade de conhecimento (inteligência cristalizada). Nos Estados Unidos ocorre o contrário, o Scholastic Assessment Test (SAT I) enfatiza mais a capacidade de raciocínio utilizando sub-testes de raciocínio verbal (analogias, completar sentenças e leitura crítica) e raciocínio matemático (problemas de aritmética, álgebra, geometria e raciocínio lógico). Um dos propósitos deste instrumento, construído na década de 40 , foi justamente a avaliação de habilidades associadas ao desempenho escolar e não a avaliação da realização escolar (Wainer, 1990).

Ao que parece o ENEM situa-se em algum lugar entre a avaliação do raciocínio e do conhecimento, mas mais próximo do primeiro que do último, como pelo menos uma pesquisa empírica evidenciou (Brito \& cols., 2000). Como já dissemos, apesar do fato de as questões do ENEM avaliarem co- nhecimento, elas o avaliam de maneira diferente das habituais provas tradicionais, privilegiando a interdisciplinaridade, o uso de situações-problema contextuadas e fornecendo informações suficientes para que o aluno encontre a solução das questões. Como os conhecimentos de diferentes áreas devem ser inter-relacionados e aplicados a situações práticas (semelhante, em certo sentido, à inteligência prática de Sternberg, Wagner, Williams \& Horvat, 1995), os esquemas habituais de solução de problemas (inteligência cristalizada) passam a não ser automaticamente suficientes, pois devem ser reorganizados em novas estratégias de solução (inteligência fluida). Acrescenta-se aí, o fato de que grande parte das informações são fornecidas nas próprias questões, exigindo a manipulação e o relacionamento simultâneo de várias informações, que como mostram alguns dos estudos recentes, é aspecto essencial da inteligência fluida (Engle, Tuholski, Laughlin \& Conway, 1999; Hunt, 1999; Primi, 1998; Primi, no prelo). Mesmo que o ENEM não tenha sido criado com o objetivo de substituir os vestibulares, gradualmente, ele vem sendo empregado como parte destes, o que determinará uma ênfase maior nos processos ligados ao raciocínio, o que caracteriza um aspecto muito positivo, embora ainda exista necessidade de aprimoramentos.

Um ponto importante é que a concepção estrutural da inteligência em cinco competências e 21 habilidades que os autores afirmam representar "as possibilidades totais da cognição humana na fase de desenvolvimento próprio aos participantes do ENEM" (INEP, 1999, p.9) não possui a abrangência que seus autores lhe atribuem. Vale ressaltar que o problema da definição das estruturas cognitivas constituintes da inteligência, tem sido a grande questão nos últimos 100 anos de inúmeros pesquisadores de pelo menos três abordagens metodológicas distintas. Embora se possa hipotetizar algumas semelhanças entre o modelo psicométrico integrado de Carroll (1993) e o modelo da matriz de competências e habilidades do ENEM (Dominar Linguagens e Compreender Fenômenos, mais próximas à inteligência cristalizada e as competências; Enfrentar Situações Problema e Construir Argumentações, mais próximas da inteligência fluida), ela está longe de representar o amplo espectro das manifestações possíveis da inteligência, descobertas pelos estudos psicométricos.

Do ponto de vista operacional, cabe um questionamento, relacionado à composição dos escores do ENEM, tendo em vista que vários itens contam simultaneamente para várias competências; o que provoca uma alta correlação entre os escores de cada competência de tal forma que, em termos práticos, as cinco competências podem não estar trazendo informações adicionais àquelas obtidas pelo escore total (soma de pontos em todos os itens de múltipla escolha). Dessa forma, é difícil sustentar a existência das cinco competências, como componentes relativamente distintos, e justificar a apresentação de escores separados. Vê-se também na construção do ENEM, uma sobrevalorização de conceitos teóricos, evidenciado pela criação das subescalas, baseadas exclusivamente na análise teórica dos itens e não a partir de uma integração de métodos empíricos e teóricos, emprega- 
dos na construção de instrumentos de avaliação que geralmente utilizam a análise fatorial dos itens para investigar a melhor maneira de combiná-los para produzir escores distintos (componentes ortogonais).

Um terceiro e último ponto a ser considerado, neste artigo, refere-se ao uso dos termos competência e habilidade. Como foi mencionado, esta nomenclatura tem sido usada por pesquisadores europeus (Perrenoud, 1997) e norte-americanos (Carroll, 1993), mas o conceito associado aos termos difere para ambos, tendo em vista que o que os europeus denominam como competência, os norte-americanos têm denominado como habilidades cognitivas.

No entanto, analisando-se as definições de competências e habilidades do ENEM, pode-se perceber que todas se referem ao domínio de determinada operação cognitiva e não ao potencial para executar a operação, o que seria mais próximo do conceito de capacidade ou habilidade, no sentido clássico dos termos. Assim, pode-se observar que tanto as definições das cinco competências, quanto das 21 habilidades referem-se tão somente a competências, isto é, níveis esperados de realização. Tal fato pode ser ilustrado a partir das definições que tomamos como exemplo: (a) dominar a norma culta da língua portuguesa (Competência I); (b) enfrentar situações-problemas (Competência III); (c) comparar processos e formação socieconômica, relacionando-os com seu contexto histórico (Habilidade 20); (d) dada uma distribuição estatística de variável social, econômica, física, química, ou biológica, traduzir e interpretar as informações disponíveis, ou reorganizá-las, objetivando interpolações ou extrapolações (Habilidade 3). Parece-nos imprescindível reduzir a confusão de nomenclaturas, sendo que uma sugestão possível seria denominar as cinco competências de 'competências gerais' e as 21 habilidades de 'competências específicas'.

Entendemos, no entanto, que os aspectos mais críticos referem-se aos dois primeiros pontos, que evidenciam a necessidade de estudos que investiguem a validade de construto do ENEM (American Educational Research Association, American Psychological Association \& National Council on Measurement in Education, 1999), o que poderia ser feito, por exemplo, por meio da análise fatorial dos itens e de estudos que correlacionassem o ENEM com outros instrumentos marcadores dos fatores definidos por Carroll (1993). Com base nas análises por nós efetuadas e em outros estudos (Brito \& cols., 2000) é provável que o ENEM se correlacione mais com medidas de inteligência fluida do que com inteligência cristalizada, se comparado com as provas tradicionais centradas no conhecimento.

Tais estudos seriam muito importantes para compreendermos exatamente quais as capacidades cognitivas avaliadas pelo ENEM, de forma a se estabelecerem as relações do conhecimento que vêm sendo descobertas à respeito dos padrões de desenvolvimento com outras variáveis como a aprendizagem, traços de personalidade, interesses profissionais, bem como, com os substratos neuro-fisiológicos da inteligência, podendo integrá-los na interpretação dos escores deste importante instrumento brasileiro de avaliação.

\section{Referências}

Ackerman, P.L. (1996). A theory of intellectual development: Process, personality, interests, and knowledge. Intelligence, 22, 227-257.

Ackerman, P.L. \& Heggestad, E.D. (1997). Intelligence, personality, and interests: Evidence for overlapping traits. Psychological Bulletin, 121, 219-245.

Ackerman, P.L., Kyllonen, P.C. \& Roberts, R.D. (1999). Learning and individual differences. Washington, DC: American Psychological Association.

American Educational Research Association, American Psychological Association \& National Council on Measurement in Education (1999). Standards for Educational and Psychological Testing. Washington, DC: American Educational Research Association

Almeida, L.S. (1988). Teorias da Inteligência. Porto: Edições Jornal de Psicologia.

Almeida, L.S. (1994) Inteligência: Definição e Medida. Aveiro: CIDInE.

Bethell-Fox, C.E., Lohman, D.F. \& Snow, R.E. (1984). Adaptative reasoning: Componential and eye movement analysis of geometric analogy performance. Intelligence, 8, 205-238.

Brito, M.R.F., Munhoz, A.M.H., Primi, R., Gonçalez, M.H., Rezi, V.C., Neves, L.F., Sanches, M.H.F. \& Marinheiro, F.B. (2000). Exames nacionais: uma análise do ENEM aplicado à matemática. Avaliação: Revista da Rede de Avaliação Institucional da Educação Superior, 5(4), 45-53.

Carroll, J.B. (1993). Human cognitive abilities: A survey of factoranalytic studies. New York: Cambridge University Press.

Engle, R.W., Tuholski, S.W., Laughlin, J.E. \& Conway, A.R.A. (1999). Working memory, short-term memory, and general fluid intelligence: A latent-variable approach. Journal of Experimental Psychology: General, 128, 309-331.

Fini, M.E., Charnet, E.M.R., Talim, S.L. \& Moraes, Z.H. (1999). Erros e acertos na elaboração de itens para a prova do ENEM: as técnicas de elaboração de itens e as questões de múltipla escolha do ENEM. Brasília: INEP.

Flanagan, D.P., Genshaft, J.L. \& Harrison, P.L. (1997). Contemporary intellectual assessment: Theories, tests, and issues. New York: The Guilford Press.

Flanagan, D.P. \& McGrew, K.S. (1997). A cross-battery approach to assessing and interpreting cognitive abilities: Narrowing the gap between practice and cognitive science. Em D.P. Flanagan, J.L. Genshaft \& P.L. Harrison (Orgs.), Contemporary intellectual assessment: Theories, tests, and issues (pp. 314-325). New York: The Guilford Press.

Goldman, S.R. \& Pellegrino, J.W. (1984). Deductions about induction: Analyses of developmental and individual differences. Em R.J. Sternberg (Org.), Advances in the psychology of human intelligence (pp. 149-197). Hillsdale NJ: LEA, Vol 2.

Guttman, L. (1954). A new approach to factor analysis: The radex. Em P.F. Lazarsfeld (Org.), Mathematical thinking in the social sciences (pp. 258-348). Glencoe, IL: Free Press.

Guilford, J.P. (1967). The nature of human intelligence. New York: McGraw-Hill.

Hambleton, H.K. \& Swaminatham, H. (1985). Item Response Theory: Principles and Applications. Boston: Kluwer. 
Horn, J.H. (1991) Measurement of intellecual capabilities: A review of theory. Em K. S. McGrew, J. K. Werder \& R. W. Woodcock WJ-R Technical Manual. (pp. 197-245). Allen, TX: DLM.

Horn, J.L. \& Noll, J. (1997). Human cognitive capabilities: Gf-Gc theory. Em D.P. Flanagan, J.L. Genshaft \& P.L. Harrison (Orgs.), Contemporary intellectual assessment: Theories, tests, and issues (pp. 53-91). New York: The Guilford Press.

Hunt, E. (1980). Intelligence as an information processing concept. British Journal of Psychology, 71, 449-474.

Hunt, E. (1996). Intelligence for the 21st Century. Trabalho apresentado na European Society for Cognitive Psychology e Spanish Society for the Study of Individual Differences, Madri, Espanha.

Hunt, E. (1999). Intelligence and human resources: Past, present and future. Em P.L. Ackerman, P.C. Kyllonen \& R.D. Roberts (Orgs.), Learning and individual differences: Process, trait and content determinants. (pp. 3-28). Washington, DC: American Psychological Association.

Instituto Nacional de Estudos e Pesquisas Educacionais (1997). Exame Nacional de Cursos: Relatório-Síntese. Brasília: INEP.

Instituto Nacional de Estudos e Pesquisas Educacionais (1998a). Exame Nacional do Ensino Médio: Relatório Final. Brasília: INEP.

Instituto Nacional de Estudos e Pesquisas Educacionais (1998b). SAEB/95: Relatório Final. Brasília: INEP.

Instituto Nacional de Estudos e Pesquisas Educacionais (1999). Exame Nacional do Ensino Médio: Documento Básico 2000. Brasília: INEP.

Kruskal, J.B. (1964). Multidimensional scaling by optimizing goodness of fit to a nonmetric hypothesis. Psychometrika, 29, 1-27.

Macedo, L. (1999). Competências e habilidades: elementos para uma reflexão pedagógica. Brasília: INEP.

Machado, L. (1999). Interdisciplinaridade e contextuação. Brasília: INEP.

Marshalek, B., Lohman, D.F. \& Snow, R.E. (1983). The complexity continuum in the radex and hierarchical models of intelligence. Intelligence, 7, 107-127.

Marañón, R.C. \& Andrés-Pueyo, A.A. (1999). El estudio de la inteligencia humana: recapitulación ante el cambio de milenio. Psicothema, 11, 453-476.

Mayer, J. \& Salovey, P. (1998) O que é inteligência emocional? Em P. Salovey \& D.J. Sluyter (Orgs.), Inteligência emocional na criança: aplicações na educação e no dia-a-dia. (pp. 1349). Rio de Janeiro: Campus.

McGrew, K.S. (1998). The intelligence test desk reference (ITDR): $G$ f-Gc cross-battery assessment. Needham Heights: Allyn \& Bacon.

Menezes, L.C., Gualtieri, R.C.E., Guimarães, R.B., Lisboa, J.C.F. \& Kawamura, M.R.D. (1999). Competência II: construir e aplicar conceitos das várias áreas do conhecimento para a compreensão de fenômenos naturais, de processos históricosgeográficos, da produção tecnológica e das manifestações artísticas. Brasília: INEP.

Newell, A. \& Simon, H.A. (1972). Human problem solving. Englewood Cliffs, NJ: Prentice-Hall.

Pellegrino, J.W. \& Glaser, R. (1979). Cognitive correlates and components in the analysis of individual differences. Intelligence, 3, 187-214.

Pellegrino, J.W. \& Lyon, D.R. (1979). The components of a com- ponential analysis. Intelligence, 3, 169-186.

Perrenoud, P. (1997). Construir as competências desde a escola. Porto Alegre: Artes Médicas.

Primi, R. (1995) Inteligência, processamento de informação e teoria da gestalt: um estudo experimental. Dissertação de Mestrado. Departamento de Pós-Graduação em Psicologia, Pontifícia Universidade Católica de Campinas, Campinas.

Primi, R. (1998). Desenvolvimento de um instrumento informatizado para avaliação do raciocínio analítico. Tese de Doutorado, Instituto de Psicologia, Universidade de São Paulo, São Paulo.

Primi, R. (no prelo) Complexity of geometric inductive reasoning tasks: Contribution to the understanding of the Fluid Intelligence. Intelligence.

Raven, J., Raven J.C. \& Court, J.H. (1998). Manual for Raven's progressive matrices and vocabulary scales: Section 1 general overview. Oxford: Oxford Psychologysts Press.

Schmid, J. \& Leiman, J.M. (1957). The development of hierarquical factor solutions. Psychometrika, 22, 53-61.

Snow, R.E. (1989). Cognitive-conative aptitude interactions in learning. Em R. Kanfer (Org.), Abilities, motivation, and methodology: The Minnesota Symposium on Learning and Individual Differences (pp. 435-474). Hillsdale: Lawrence Erlbaum Associates.

Snow, R.E. (1992). Aptitude theory: Yesterday, today and tomorrow. Educational Psychologist, 27, 5-32.

Snow, R.E., Kyllonen P.C. \& Marshalek B. (1984). The topography of ability and learning correlations. Em R.J. Sternberg (Org.), Advances in the psychology of human intelligence (pp. 47-103). Vol. 2. Hillsdale, NJ: Lawrence Erlbaum Associates.

Spearman, C. (1927). The abilities of man: Their nature and measurement. New York: Macmillan.

Sternberg, R.J. (1977). A component process in analogical reasoning. Psychological Review, 84, 353-378.

Sternberg, R.J. (1979). The nature of mental abilities. American Psychologist, 34, 214-230.

Sternberg, R.J. (1980). Factor theories of intelligence are all right almost. Educational Researcher, 9, 6-13.

Sternberg, R.J. (1981). The evolution of theories of intelligence. Intelligence, 5, 209-230.

Sternberg, R.J. (1983). Components of human intelligence. Cognition, $15,1-48$.

Sternberg, R.J. (1986). Toward a unified theory of human reasoning. Intelligence, 10, 281-314.

Sternberg, R.J., Wagner, R.K., Williams, W.M. \& Horvat, J.A. (1995). Testing common sense. American Psychologist, 50, 912 927.

Thurstone, L.L. (1938). Primary mental abilities. Chicago: University Chicago Press.

Wainer, H. Ed. (1990). Computerized adaptive testing: A primer. Hillsdale: Lawrence Erlbaum Associates.

Wechsler, D. (1964). WISC - Escala de inteligência Wechsler para crianças. (A.M. Poppovic, Trad.). São Paulo: CEPA.

Recebido em 25.10.2000

Primeira decisão editorial em 05.06.2001

Versão final em 05.07.2001

Aceito em 19.07.2001 